\title{
High tunnel magnetoresistance in spin-polarized scanning tunneling microscopy of Co nanoparticles on $\mathrm{Pt}(111)$
}

\author{
S. Rusponi, ${ }^{a)}$ N. Weiss, T. Cren, M. Epple, and H. Brune \\ Institut de Physique des Nanostructures, Ecole Polytechnique Fédérale de Lausanne (EPFL), \\ CH-1015 Lausanne, Switzerland
}

(Received 11 April 2005; accepted 9 August 2005; published online 14 October 2005)

\begin{abstract}
We employ variable-temperature spin-polarized scanning tunneling microscopy in constant current mode to read the magnetic state of monodomain cobalt nanoparticles on $\mathrm{Pt}(111)$. In order to avoid stray fields we use in situ prepared antiferromagnetically $(\mathrm{Cr})$ coated $\mathrm{W}$ tips. The contrast in apparent height between nanoparticles with opposite magnetization is typically $\Delta z=0.20 \pm 0.05 \AA$, but can reach up to $1.1 \AA$, indicating $80 \%$ spin-polarization of the nanoparticles and $850 \%$ magnetoresistance of the tip-sample tunnel junction with tip and sample at $300 \mathrm{~K}$ and $160 \mathrm{~K}$, respectively. There is no zero-bias anomaly. These results suggest state-selective tunneling which is expected to lead to very high magnetoresistance values. (C) 2005 American Institute of Physics.

[DOI: $10.1063 / 1.2077856]$
\end{abstract}

The recent development of spin-polarized scanning tunneling microscopy (SP-STM) $)^{1-3}$ has provided a tool to investigate magnetism in real space down to the atomic scale. ${ }^{4}$ It is based on a spin-polarized tunnel current flowing across the vacuum barrier between a magnetic tip and sample..$^{2,3,5,6}$ To date, the prevailing operation modes of SP-STM are the spectroscopic mode (SP-STS) and the differential magnetic mode. In the first case, one records maps of the differential conductivity $d I / d V(V)$, revealing the convoluted tip-sample local density of states (LDOS) at an energy $e V$ with respect to $E_{F}{ }^{1}$ In the second case, the magnetization of the tip is periodically modulated to strictly separate the magnetic information of a sample surface from its topography. ${ }^{2}$

Magnetic contrast on individual nanoparticles was observed by means of low-temperature (15 K) SP-STS with ferromagnetically coated $\mathrm{W}$ tips ${ }^{7,8}$ or by using noninvasive antiferromagnetic tips, both bulk ${ }^{9}$ and coated ones. ${ }^{10-12}$ Room-temperature SP-STS has been demonstrated on Fe islands antiferromagnetically exchanged coupled with the $\mathrm{Cr}(001)$ substrate. ${ }^{13}$ Very few attempts were done to gain information on the processes involved during spin tunneling. ${ }^{12,14,15}$ For example, a complicate interplay between structural and magnetic properties was recently observed by SP-STS on Co islands on $\mathrm{Cu}(111) .{ }^{12}$ However, this spin resolved information is fundamental for developing nanoscale spintronics devices.

In this letter, we demonstrate magnetic contrast on individual Co nanoparticles on Pt(111) with an SP-STM operated in constant current mode, as in Ref. 5, and at variable sample temperature with the Cr-coated $\mathrm{W}$ tip being close to room temperature. Two monolayer high Co islands on $\mathrm{Pt}(111)$ with perpendicular magnetization (up and down) are discerned by a difference in apparent height of typically $\Delta z=0.20 \AA$. The maximum magnetic contrast was $\Delta z=1.1 \AA$ indicative of $80 \%$ spin polarization of the tip-sample tunnel junction. This implies a minimum spin polarization of the Co nanoparticles of $80 \%$, far beyond the values reported for Co bulk and thin films. ${ }^{16,17}$ In addition, this difference in the apparent height is equivalent to a tunneling magnetoresistance (TMR) of $850 \%$,

${ }^{a}$ Electronic mail: stefano.rusponi@epfl.ch value observed with the tip temperature close to $300 \mathrm{~K}$, and the sample just below the nanoparticles blocking temperature $(160 \mathrm{~K})$. Artifacts of structural origin ${ }^{12}$ were excluded by verifying that all the islands have the same apparent height when imaged with a bare $\mathrm{W}$ tip.

STM measurements were performed in an ultrahigh vacuum (UHV) chamber (base pressure $3 \times 10^{-11}$ mbar) designed for carrying out molecular-beam epitaxy, variable temperature STM, and magneto-optical Kerr effect (MOKE) measurements at a single sample position. ${ }^{18}$ During STM measurements at low sample temperatures, the STM head and tip stay close to room temperature $(\approx 280 \mathrm{~K}),{ }^{19}$ since they are thermally weakly coupled to the sample and fully exposed to the ambient radiation. $\mathrm{Cr}$ has been deposited on the W tip from an evaporator consisting of a resistively heated W filament carrying a high-purity bar of $\mathrm{Cr}(99.95 \%)$. The tip coating is freshly prepared every day. In situ $\mathrm{Ar}^{+}$-ion sputtering and field emission were used to clean the tip apex prior to deposition. Co has been evaporated onto the clean $\mathrm{Pt}(111)$ sample from a carefully outgassed Co rod (99.995\%) at $8 \times 10^{-11}$ mbar background pressure.

Co double-layer islands were created by deposition of 0.40 monolayers (ML) on the Pt(111) substrate held at $130 \mathrm{~K}$ and subsequent annealing to $340 \mathrm{~K} .{ }^{18}$ The $-9.4 \%$ misfit between $\mathrm{Co}$ and $\mathrm{Pt}$ leads to partial dislocations in the first layer, ${ }^{20}$ in double-layer islands, the stress is partly relieved by a moiré structure (Fig. 1). ${ }^{21,22}$ This moiré pattern causes irregular heights in individual line scans which can be reduced by averaging over a few line scans [Fig. 1(b)]. Imaged with a W tip, the islands have a uniform apparent height of $4.15 \pm 0.05 \AA$, close to the distance of two atomic Cohexagonal-close-packed planes (4.07 $\AA$ ).

The STM image acquired in constant current mode with a Cr-coated tip (Fig. 2) clearly shows magnetic contrast, i.e., there are two island species characterized by apparent heights differing by $\Delta z=0.20 \pm 0.05 \AA$. Artifacts due to changes of the tip state are excluded by the correlation in island height between subsequent line scans and by the fact that selected islands reproducibly appear with their characteristic heights. Note that in addition to the spin contrast, the absolute island heights also differ from the ones of Fig. 1 
(a)

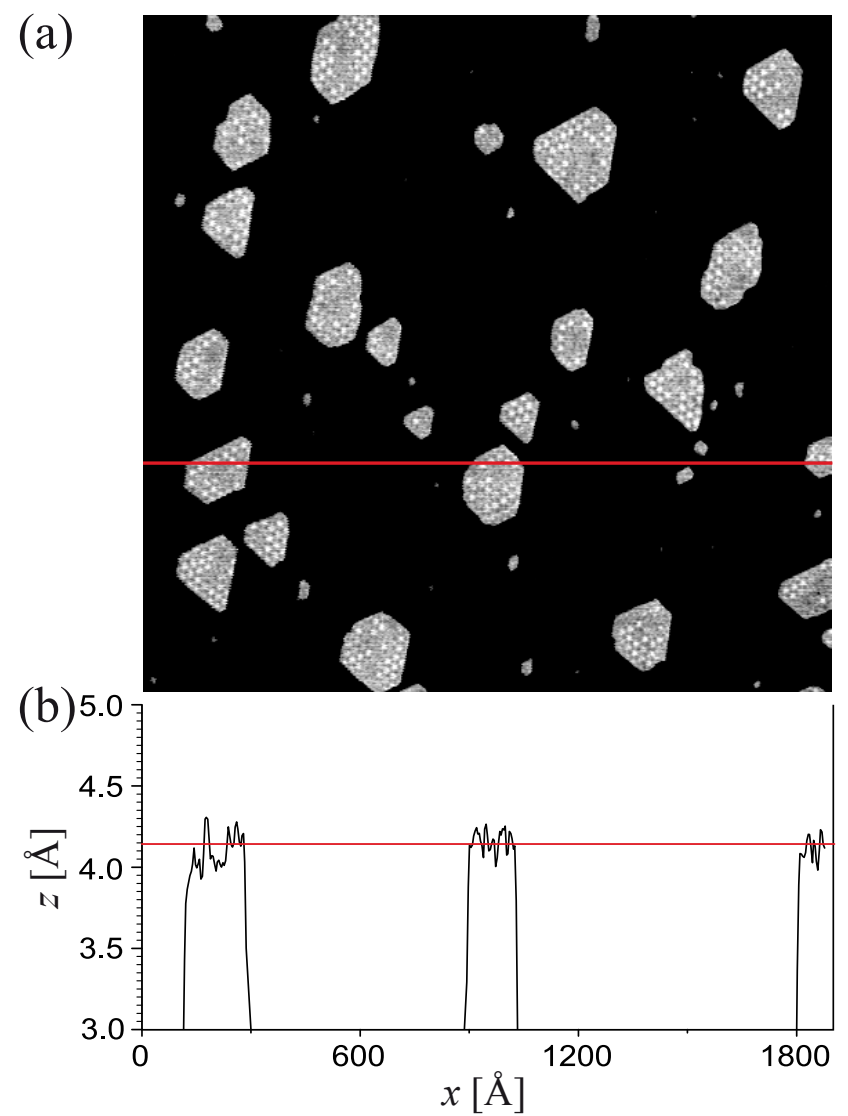

(b) 5.0

FIG. 1. (Color online) (a) Constant current STM image of double-layer Co islands recorded with a bare $\mathrm{W}$ tip. The moiré structure is caused by the lattice misfit and resolved with a corrugation in apparent height of $0.30 \pm 0.03 \AA\left(T_{\text {sample }}=140 \mathrm{~K}, V_{t}=-0.2 \mathrm{~V}\right.$, and $\left.I_{t}=1.0 \mathrm{nA}\right)$. (b) Constant cur rent profile obtained by averaging over \pm 5 line scans around the position marked in (a). All islands appear with identical heights irrespective of their magnetization (absence of spin contrast).

since the overall LDOS at $E_{F}$, and presumably the work function of the tip, $\phi_{t}$, are modified by the $\mathrm{Cr}$ coating. Approximately one-half of the islands are imaged dark and the other half bright, corresponding to a vanishing net magnetization of our sample, as expected from the sample being zero-field cooled. Spin contrast was only observed for sample temperatures below $160 \mathrm{~K}$, and for a thickness of the $\mathrm{Cr}$ coating ranging from 20 to 40 MLs. This coating thickness range has been reported to yield out-of-plane sensitivity. ${ }^{10}$ We therefore conclude that the islands have outof-plane easy axis and blocking temperatures above $160 \mathrm{~K}$. Both observations are in excellent agreement with spatially integrating measurements by in situ MOKE. ${ }^{18}$ We kept the tunneling current below $1.0 \mathrm{nA}$ since higher values produced a fast degradation of the spin contrast. The bias voltage was typically in the range $\pm 0.2 \mathrm{~V}$, however, different bias values $( \pm 0.6 \mathrm{~V})$ were also applied without affecting the spin contrast.

The tunneling current can be separated in an unpolarized part $I_{0}$, given by the spin integrated LDOS of the sample at the tip position, and a spin-polarized contribution $I_{s}$, corresponding to the projection of the vector of the energy integrated spin-polarized LDOS of the sample onto the one of the tip. ${ }^{23}$ The tunneling current is then $I_{p}=I_{0}+I_{s}$ for parallel magnetization of tip and sample and $I_{a}=I_{0}-I_{s}$ for the antiparallel configuration. In constant current STM images, this implies higher and lower apparent heights, $z \pm \Delta z / 2$. The po- (a)

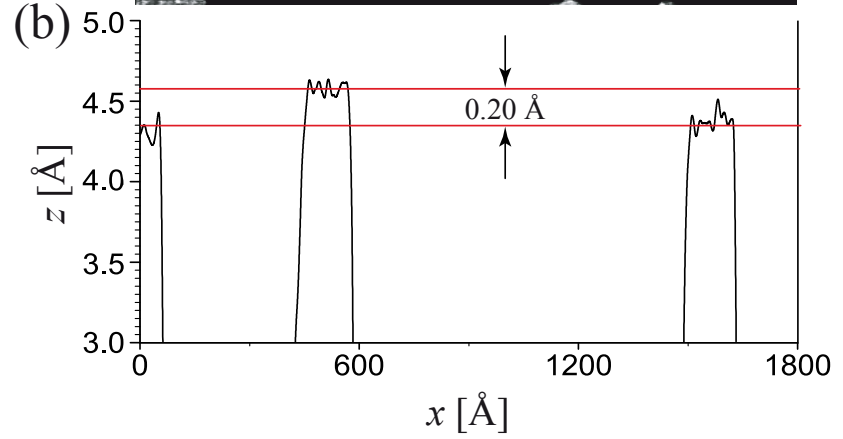

FIG. 2. (Color online) (a) Constant current STM image of double-layer Co islands recorded with a $\mathrm{Cr}$-coated $\mathrm{W}$ tip. Islands with opposite magnetization appear with two different heights $\left(T_{\text {sample }}=140 \mathrm{~K}, T_{\text {tip }} \approx 280 \mathrm{~K}, V_{t}=\right.$ $-0.08 \mathrm{~V}, I_{t}=0.3 \mathrm{nA}$ ). (b) Averaged ( \pm 5 lines) profile at the indicated position in (a) showing a difference of $0.20 \pm 0.05 \AA$ in the apparent height of islands with opposite magnetization (spin contrast).

larization of the tunneling junction $P=\left(I_{p}-I_{a}\right) /\left(I_{p}+I_{a}\right)$ is given by: ${ }^{5}$

$$
P=\frac{I_{s}}{I_{0}}=\frac{\exp (A \sqrt{\phi} \Delta z)-1}{\exp (A \sqrt{\phi} \Delta z)+1}
$$

where $\phi$ is the average over the work functions of tip and sample and $A=2 \sqrt{2 m_{e} / \hbar^{2}}=1.025 \mathrm{eV}^{-1 / 2} \AA^{-1}$. Assuming a typical value of $\phi=4 \mathrm{eV}$, we find $P=0.20 \pm 0.05$ from the difference in apparent height of Fig. 2.

The spin contrast strongly depends on the spin polarization and on the orientation of the magnetic moment of the atoms at the tip apex. These properties are expected to change for each newly prepared tip. Accordingly, we observed different $\Delta z$-values from day to day, $0.2 \AA$ being the typical value. In a single case, we observed a difference of $\Delta z=1.1 \pm 0.1 \AA$ (Fig. 3), corresponding to $P=0.80 \pm 0.04$. The polarization of the tunneling junction is first-order linked to the tip and sample polarizations by the relation $P \sim P_{T} P_{S} \cos \theta$, where $\theta$ is the angle between the tip and sample magnetization. ${ }^{24}$ The previous relation implies a Co polarization of at least $80 \%$, about two times larger than the value determined for Co bulk by Andreev reflection. ${ }^{16,17}$

From the observed apparent height differences $\Delta z$, we can derive the TMR for the case of a tunnel junction formed by the tip kept at constant height above an island which magnetization switches between up and down

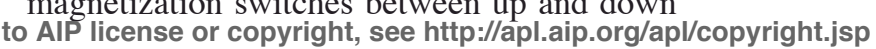


(a)

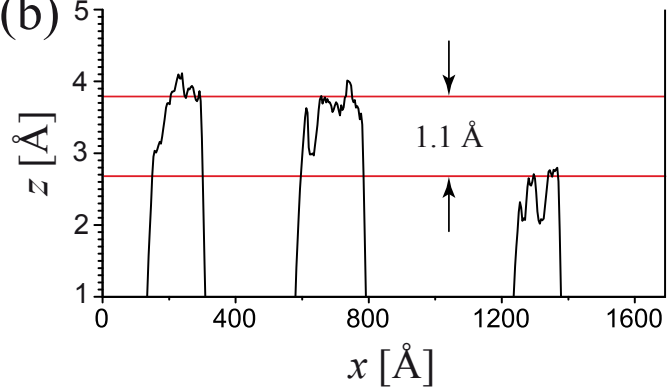

FIG. 3. (Color online) (a) Constant current STM image of double-layer Co islands recorded with a Cr-coated W tip $\left(T_{\text {sample }}=140 \mathrm{~K}, T_{\text {tip }} \approx 280 \mathrm{~K}, V_{t}=\right.$ $\left.-0.2 \mathrm{~V}, I_{t}=0.3 \mathrm{nA}\right)$. (b) Averaged ( \pm 5 lines) line scan at the indicated position in (a). A surprisingly high difference of $1.1 \pm 0.1 \AA$ in the apparent height of islands with opposite magnetization is observed.

$$
\Delta R / R=\frac{R_{a}-R_{p}}{R_{p}}=\frac{I_{p}}{I_{a}}-1=\exp (A \sqrt{\phi} \Delta z)-1 .
$$

For Figs. 2 and 3, Eq. (2) gives $\Delta R / R=50 \pm 15 \%$ and $\Delta R / R=850 \pm 200 \%$, respectively. The latter value largely overcomes the highest TMR value yet reported of about $200 \%$ at room temperature with $\mathrm{MgO}$ tunnel barrier. ${ }^{25-27}$

Our results can be rationalized considering the following arguments. First, it has been demonstrated that TMR strongly depends on the electronic band structure of the two electrodes. ${ }^{28}$ Spin-polarized surface states are known to increase the vacuum-tunneling magnetoresistance, ${ }^{29}$ and giant TMR values, in excess of $1000 \%$, have been predicted for junction dominated by state-selective tunneling. ${ }^{30,31}$ For ideal planar Co(0001) electrodes separated by a vacuum gap, calculations based on the density-functional theory show a TMR of $260 \% .{ }^{32}$ In supported two-dimensional nanostructures the band narrowing due to the reduced symmetry and the polarization of the substrate could lead to even higher TMR value. Second, SP-STM experiments under UHV conditions warrant the absence of inelastic scattering from impurities or defects at the interfaces and in the amorphous barrier. ${ }^{14,29}$ Consequently, with respect to oxide-based tunnel junctions, we expect higher TMR values and the absence of zero-bias anomaly, ${ }^{14,32}$ as we experimentally observed up to bias voltages of $\pm 0.6 \mathrm{~V}$.

Freshly prepared tips gave magnetic contrast with a probability of about $25 \%$. A fast degradation of the magnetic contrast was also observed after about 20 images. This relatively low success rate is probably caused by the poor control of the Néel temperature $T_{N}$ of the $\mathrm{Cr}$ coating. In thin films with a thickness larger than $20 \mathrm{ML}$, the Néel temperature is expected to approach the bulk value of $311 \mathrm{~K} .{ }^{33}$ However, $T_{N}$ of the tip apex depends on its exact atomic conformation and on occasional adsorbed impurities that can cause $T_{N}$ to be lower than the tip temperature.

${ }^{1}$ M. Bode, M. Getzlaff, and R. Wiesendanger, Phys. Rev. Lett. 81, 4256 (1998).

${ }^{2}$ W. Wulfhekel and J. Kirschner, Appl. Phys. Lett. 75, 1944 (1999).

${ }^{3}$ M. Bode, Rep. Prog. Phys. 66, 523 (2003).

${ }^{4}$ S. Heinze, M. Bode, A. Kubetzka, O. Pietzsch, X. Nie, S. Blügel, and R. Wiesendanger, Science 288, 1805 (2000).

${ }^{5}$ R. Wiesendanger, H.-J. Güntherodt, G. Güntherodt, R. Gambino, and R. Ruf, Phys. Rev. Lett. 65, 247 (1990).

${ }^{6}$ S. F. Alvarado and P. Renaud, Phys. Rev. Lett. 68, 1387 (1992).

${ }^{7}$ A. Kubetzka, O. Pietzsch, M. Bode, and R. Wiesendanger, Phys. Rev. B 63, 140407(R) (2001).

${ }^{8}$ A. Yamasaki, W. Wulfhekel, R. Hertel, S. Suga, and J. Kirschner, Phys. Rev. Lett. 91, 127201 (2003).

${ }^{9}$ N. Berdunov, S. Murphy, G. Mariotto, and I. V. Shvets, Phys. Rev. Lett. 93, 057201 (2004).

${ }^{10}$ A. Kubetzka, M. Bode, O. Pietzsch, and R. Wiesendanger, Phys. Rev. Lett. 88, 057201 (2002).

${ }^{11}$ M. Bode, O. Pietzsch, A. Kubetzka, and R. Wiesendanger, Phys. Rev. Lett. 92, 067201 (2004).

${ }^{12}$ O. Pietzsch, A. Kubetzka, M. Bode, and R. Wiesendanger, Phys. Rev. Lett. 92, 057202 (2004).

${ }^{13}$ R. Ravlić, M. Bode, and R. Wiesendanger, J. Phys.: Condens. Matter 15, S2513 (2003).

${ }^{14}$ H. F. Ding, W. Wulfhekel, J. Henk, P. Bruno, and J. Kirschner, Phys. Rev. Lett. 90, 116603 (2003).

${ }^{15}$ S. Okuno, T. Kishi, and K. Tanaka, Phys. Rev. Lett. 88, 066803 (2002).

${ }^{16}$ R. J. Soulen Jr., J. M. Byers, M. S. Osofsky, B. Nadgorny, T. Ambrose, S. F. Cheng, P. R. Broussard, C. T. Tanaka, J. Nowak, J. S. Moodera, A. Barry, and J. M. D. Coey, Science 282, 85 (1998).

${ }^{17}$ S. K. Upadhyay, A. Palanisami, R. N. Louie, and R. A. Buhrman, Phys. Rev. Lett. 81, 3247 (1998).

${ }^{18}$ S. Rusponi, T. Cren, N. Weiss, M. Epple, P. Buluschek, L. Claude, and H. Brune, Nat. Mater. 2, 546 (2003).

${ }^{19}$ M. Bott, T. Michely, and G. Comsa, Rev. Sci. Instrum. 66, 4135 (1995).

${ }^{20}$ T. Cren, S. Rusponi, N. Weiss, M. Epple, and H. Brune, J. Phys. Chem. B 108, 14685 (2004).

${ }^{21}$ P. Grütter and U. T. Dürig, Phys. Rev. B 49, 2021 (1994).

${ }^{22}$ E. Lundgren, B. Stanka, M. Schmid, and P. Varga, Phys. Rev. B 62, 2843 (2000).

${ }^{23}$ D. Wortmann, S. Heinze, P. Kurz, G. Bihlmayer, and S. Blügel, Phys. Rev. Lett. 86, 4132 (2001).

${ }^{24}$ T. Miyazaki and N. Tezuka, J. Magn. Magn. Mater. 139, L231 (1995); J. Slonczewski, Phys. Rev. B 39, 6995 (1989).

${ }^{25}$ S. P. Parkin, C. Kaiser, A. Panchula, P. M. Rice, B. Hughes, M. Samant, and S.-H. Yang, Nat. Mater. 3, 862 (2004).

${ }^{26}$ S. Yuasa, T. Nagahama, A. Fukushima, Y. Suzuki, and K. Ando, Nat. Mater. 3, 868 (2004).

${ }^{27}$ D. D. Djayaprawira, K. Tsunekawa, M. Nagai, H. Maehara, S. Yamagata, N. Watanabe, S. Yuasa, Y. Suzuki, and K. Ando, Appl. Phys. Lett. 86, 092502 (2005)

${ }^{28}$ P. LeClair, J. T. Kohlhepp, C. H. van de Vin, H. Wieldraaijer, H. J. M. Swagten, W. J. M. de Jonge, A. H. Davis, J. M. MacLaren, J. S. Moodera, and R. Jansen, Phys. Rev. Lett. 88, 107201 (2002).

${ }^{29}$ R. Wiesendanger, M. Bode, and M. Getzlaff, Appl. Phys. Lett. 75, 124 (1999).

${ }^{30}$ J. Mathon and A. Umerski, Phys. Rev. B 63, 220403 (2001).

${ }^{31}$ X.-G. Zhang and W. H. Butler, Phys. Rev. B 70, 172407 (2004).

${ }^{32}$ J. Henk and P. Bruno, Phys. Rev. B 68, 174430 (2003).

${ }^{33}$ H. Zabel, J. Phys.: Condens. Matter 11, 9303 (1999). 